\title{
Selection of Cementite Variants Precipitated under Magnetic Field in Ferrite Matrix of an Fe-C Alloy
}

\author{
Hui-jin CHOE, ${ }^{1)}$ Tomoyuki TERAI, ${ }^{1) *}$ Izuru MIYAZAKI, ${ }^{2)}$ Sukeyoshi YAMAMOTO, ${ }^{3)}$ Mitsuharu YONEMURA, ${ }^{3)}$ \\ Takashi FUKUDA ${ }^{1)}$ and Tomoyuki KAKESHITA ${ }^{11}$
}

1) Department of Materials Science and Engineering, Graduate School of Engineering, Osaka University, 2-1 Yamadaoka, R\&D Labs., Inc., Yokomichi 41-1, Nagakude, Aichi, 480-1192 Japan.

3) Nippon Steel \& Sumitomo Metal Corporation, 1-8 Fuso-cho, Amagasaki, Hyogo Prefecture, 660-0891 Japan.

(Received on March 23, 2016; accepted on May 27, 2016)

\begin{abstract}
We have studied the influence of magnetic field on precipitation of cementite $\left(\mathrm{Fe}_{3} \mathrm{C}\right)$ using an $\mathrm{Fe}-0.01 \mathrm{C}$ (mass\%) alloy. Among twelve variants of cementite formed at $473 \mathrm{~K}$ in the ferrite ( $\alpha$-phase) matrix, the fraction of variants with the highest magnetocrystalline anisotropy energy decreases by the application of a magnetic field of $7 \mathrm{~T}$. In addition, the total number of particles of cementite decreases after the application of a magnetic field. These results implies that microstructure of $\mathrm{Fe}-\mathrm{C}$ alloys can be controlled by magnetic field.
\end{abstract}

KEY WORDS: iron-carbon alloys; texture control; magnetic anisotropy; shape anisotropy.

\section{Inrotduction}

Application of magnetic field is one of effective methods for controlling the microstructure of alloys and compounds. The magnetic field is especially effective at controlling microstructures in alloys with a ferromagnetic matrix or precipitate. Such examples can be found in martensitic transformation $^{1,2)}$ in some iron-based alloys, precipitation of nitride in $\mathrm{Fe}-\mathrm{N}$ alloys, ${ }^{3,4)}$ disorder-order transformations in $\mathrm{Co}-\mathrm{Pt}$ alloys ${ }^{5)}$ and $\mathrm{Fe}-\mathrm{Pd}$ alloys, ${ }^{6-8)}$ recrystallization in Fe-35Co alloys, ${ }^{9}$ and grain growth in $\mathrm{Fe}-\mathrm{Si}$ alloys. ${ }^{10}$ )

From an engineering point of view, the effect of the magnetic field is especially important in phase transformations in steels. It has been reported based on thermodynamic considerations that the application of a magnetic field significantly affects the phase diagrams of the binary $\mathrm{Fe}-\mathrm{C}$ system $^{11,12)}$ and $\mathrm{Fe}-\mathrm{Fe}_{3} \mathrm{C}$ system. ${ }^{13)}$ In addition, the influence of the magnetic field on the morphologies of various phases such as proeutectoid ferrite, ${ }^{14-16)}$ pearlite ${ }^{17,18)}$ and proeutectoid cementite ${ }^{19,20)}$ has been investigated. However, the influence of the magnetic field on the formation of cementite is not yet well understood, although cementite is the most important carbide in $\mathrm{Fe}-\mathrm{C}$ alloys.

Among various formation processes of cementite, the simplest one would be the precipitation of cementite from the ferrite phase in a carbon-supersaturated $\mathrm{Fe}-\mathrm{C}$ alloy. The present study is motivated to clarify the influence of magnetic fields on this process.

Cementite has an orthorhombic crystal structure with Pnma space group. ${ }^{21,22)}$ The lattice parameters are $a=$

\footnotetext{
* Corresponding author: E-mail: terai@mat.eng.osaka-u.ac.jp DOI: http://dx.doi.org/10.2355/isijinternational.ISIJINT-2016-144
}

$0.5090, b=0.6744$, and $c=0.4525 \mathrm{~nm}^{21)}$ Twelve variants of cementite are naturally introduced when it forms in a ferrite matrix with cubic structure. We label them V1 to V12. The orientation relationships between the ferrite $(\alpha)$ and the twelve variants of cementite $(\theta)$ are shown in Table 1. The shape of cementite precipitated in ferrite matrix is plate-like. ${ }^{23)}$ Each plate is elongated in the $[100]_{\theta}$ direction and consists of fine needles as shown in Fig. 1(a). Cementite is ferromagnetic up to its Curie temperature $\left(T_{\mathrm{c}}\right)$ of $484 \mathrm{~K}$, and the easy axis of magnetization is the $c$-axis $\left([001]_{\theta}\right){ }^{24)}$

Because of the shape and magnetocrystalline anisotropy of cementite, we may expect that the fraction of the twelve variants will be influenced by the application of a magnetic field. If the shape anisotropy plays an essential role, the variants with their long axes aligned in the field direction will be formed preferentially compared to other variants. Otherwise, if the magnetocrystalline anisotropy plays an essential role, the variants with their easy axes aligned in the field direction will be formed preferentially.

\section{Precipitation of Cementite from Carbon-supersatu- rated Ferrite}

In the present study, we employed an $\mathrm{Fe}-0.01$ mass\% $\mathrm{C}$ alloy to clarify the influence of the magnetic field on the precipitation of cementite. An ingot of the Fe-0.01 mass\% $\mathrm{C}$ alloy was prepared by arc melting and hot-rolled at 1473 $\mathrm{K}$ to produce several rods. The rods were heat-treated at $1273 \mathrm{~K}$ for $24 \mathrm{~h}$ for homogenization. The rods were then elongated by $3 \%$ and subjected to strain-annealing at 1073 $\mathrm{K}$ as indicated in Fig. 2 to obtain single crystals of the ferrite ( $\alpha$-phase). Two single crystals (Specimen-I, Specimen-II) of the $\alpha$-phase were cut from the rods. Specimen-I has three 
Table 1. Orientation relationship between 12 variants of cementite $(\theta)$ and ferrite $(\alpha)$. And the angle between the long axis of cementite and the field direction $\left(\theta_{1}\right)$, and that between the easy axis of cementite and field direction $\left(\theta_{2}\right)$ for the 12 variants. A to $G$ are marks used to indicate the orientation of traces (Fig. 1).

\begin{tabular}{|c|c|c|c|c|c|c|c|c|c|c|}
\hline \multirow[b]{2}{*}{ Variant } & \multirow[b]{2}{*}[100]{$_{\theta}$} & \multirow[b]{2}{*}[010]{$_{\theta}$} & \multirow[b]{2}{*}[001]{$_{\theta}$} & \multicolumn{3}{|c|}{ Specimen-I } & \multicolumn{4}{|c|}{ Specimen-II } \\
\hline & & & & $\begin{array}{l}\text { Trace on }(1 \overline{10})_{\alpha}, \\
\text { mark }\end{array}$ & $\theta_{1}\left({ }^{\circ}\right)$ & $\theta_{2}\left(^{\circ}\right)$ & $\begin{array}{r}\text { Trace on }( \\
\text { mark }\end{array}$ & 01) $\alpha$ & $\theta_{1}\left(^{\circ}\right)$ & $\theta_{2}\left({ }^{\circ}\right)$ \\
\hline V1 & {$[111]_{\alpha}$} & {$[\overline{1} \overline{1} 2]_{\alpha}$} & {$[1 \overline{1} 0]_{\alpha}$} & - & 0 & 90 & {$[110]_{\alpha}$} & $\mathbf{E}$ & 35.3 & 90 \\
\hline V2 & {$[111]_{\alpha}$} & {$[\overline{2} 11]_{\alpha}$} & {$[0 \overline{1} 1]_{\alpha}$} & {$[\overline{1} \overline{1} \overline{1}]_{\alpha} \quad \mathbf{B}$} & 0 & 90 & {$[100]_{\alpha}$} & $\mathbf{F}$ & 35.3 & 60 \\
\hline V3 & {$[111]_{\alpha}$} & {$[1 \overline{2} 1]_{\alpha}$} & {$[\overline{1} 01]_{\alpha}$} & {$[\overline{1} \overline{1} \overline{1}]_{\alpha} \quad \mathbf{B}$} & 0 & 90 & {$[0 \overline{1} 0]_{\alpha}$} & D & 35.3 & 60 \\
\hline V4 & {$[1 \overline{1} 1]_{\alpha}$} & {$[\overline{1} 12]_{\alpha}$} & {$[\overline{1} \overline{1} 0]_{\alpha}$} & {$[00 \overline{1}]_{\alpha} \quad \mathbf{A}$} & 70.5 & 35.3 & {$[1 \overline{1} 0]_{\alpha}$} & G & 90 & 0 \\
\hline V5 & {$[1 \overline{1} 1]_{\alpha}$} & {$[121]_{\alpha}$} & {$[\overline{1} 01]_{\alpha}$} & {$[\overline{1} \overline{1} \overline{1}]_{\alpha} \quad \mathbf{B}$} & 70.5 & 90 & {$[010]_{\alpha}$} & D & 90 & 60 \\
\hline V6 & {$[1 \overline{1} 1]_{\alpha}$} & {$[\overline{2} \overline{1} 1]_{\alpha}$} & {$[011]_{\alpha}$} & {$[\overline{1} \overline{1} 1]_{\alpha} \quad \mathbf{C}$} & 70.5 & 35.3 & {$[\overline{1} 00]_{\alpha}$} & $\mathbf{F}$ & 90 & 60 \\
\hline V7 & {$[\overline{1} \overline{1} 1]_{\alpha}$} & {$[112]_{\alpha}$} & {$[\overline{1} 10]_{\alpha}$} & - & 70.5 & 90 & {$[\overline{1} \overline{1} 0]_{\alpha}$} & $\mathbf{E}$ & 35.3 & 90 \\
\hline V8 & {$[\overline{1} \overline{1} 1]_{\alpha}$} & {$[2 \overline{1} 1]_{\alpha}$} & {$[011]_{\alpha}$} & {$[\overline{1} \overline{1} 1]_{\alpha} \quad \mathbf{C}$} & 70.5 & 35.3 & {$[\overline{1} 00]_{\alpha}$} & $\mathbf{F}$ & 35.3 & 60 \\
\hline V9 & {$[\overline{1} \overline{1} 1]_{\alpha}$} & {$[\overline{1} 21]_{\alpha}$} & {$[101]_{\alpha}$} & {$[\overline{1} \overline{1} 1]_{\alpha} \quad \mathbf{C}$} & 70.5 & 35.3 & {$[010]_{\alpha}$} & D & 35.3 & 60 \\
\hline V10 & {$[\overline{1} 11]_{\alpha}$} & {$[1 \overline{1} 2]_{\alpha}$} & {$[110]_{\alpha}$} & {$[001]_{\alpha} \quad \mathbf{A}$} & 70.5 & 35.3 & {$[\overline{1} 10]_{\alpha}$} & $\mathbf{G}$ & 90 & 0 \\
\hline V11 & {$[\overline{1} 11]_{\alpha}$} & {$[\overline{1} 21]_{\alpha}$} & {$[101]_{\alpha}$} & {$[\overline{1} \overline{1} 1]_{\alpha} \quad \mathbf{C}$} & 70.5 & 35.3 & {$[010]_{\alpha}$} & D & 90 & 60 \\
\hline V12 & {$[\overline{1} 11]_{\alpha}$} & {$[211]_{\alpha}$} & {$[0 \overline{1} 1]_{\alpha}$} & {$[\overline{1} \overline{1} \overline{1}]_{\alpha} \quad \mathbf{B}$} & 70.5 & 90 & {$[100]_{\alpha}$} & $\mathbf{F}$ & 90 & 60 \\
\hline
\end{tabular}

(a)
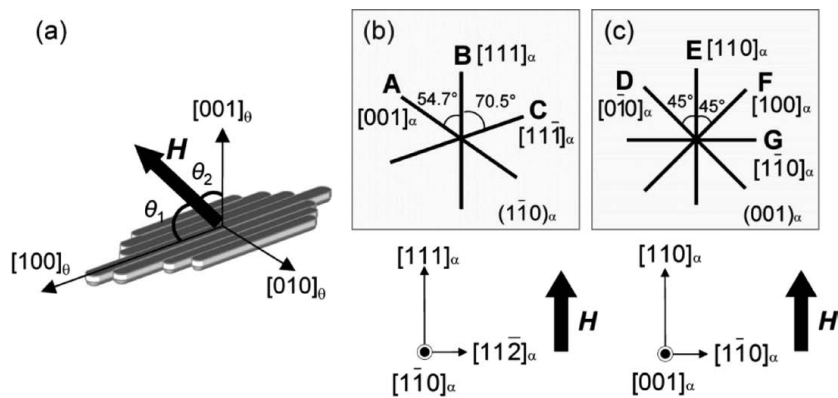

Fig. 1. The morphology of cementite (a) and traces of cementite on the $(110)_{\alpha}$ plane (b) and on the $(001)_{\alpha}$ plane. $\theta_{1}$ is the angle between field direction $(H)$ and the long axis of cementite. $\theta_{2}$ is the angle between field direction $(\boldsymbol{H})$ and easy axis of cementite.

edges parallel to the $[111]_{\alpha},[11 \overline{2}]_{\alpha}$, and $[1 \overline{1} 0]_{\alpha}$ directions; we apply magnetic field in the $[111]_{\alpha}$ direction, which is parallel to the elongated direction of cementite for several variants (V1, V2 and V3 in Table 1). Specimen-II has three edges parallel to $[110]_{\alpha},[1 \overline{1} 0]_{\alpha}$, and $[100]_{\alpha}$ directions; we apply magnetic field in the $[110]_{\alpha}$ direction, which is parallel to the easy axis of cementite for several variants (V4 and V10 in Table 1). The size of the two specimens was $5 \times 3 \times 1$ $\mathrm{mm}$. The two specimens were solution heat-treated at 1073 $\mathrm{K}$ for $2 \mathrm{~h}$, and then quenched in ice water. The precipitation of cementite under magnetic fields of $0 \mathrm{~T}$ and $7 \mathrm{~T}$ was done during a heat-treatment at $473 \mathrm{~K}$, which is $11 \mathrm{~K}$ below the Curie temperature $\left(T_{\mathrm{c}}=484 \mathrm{~K}\right)$ of cementite as shown in Fig. 2. The heating and the cooling rates were both $10 \mathrm{~K} / \mathrm{min}$, and the holding time was approximately $24 \mathrm{~h}$. The microstructure of the heat-treated specimens was observed from on the $(1 \overline{1} 0)_{\alpha}$ of Specimen-I and the $(001)_{\alpha}$ of Specimen-II using a scanning electron microscope (SEM).

As cementite has an elongated plate-like shape and its

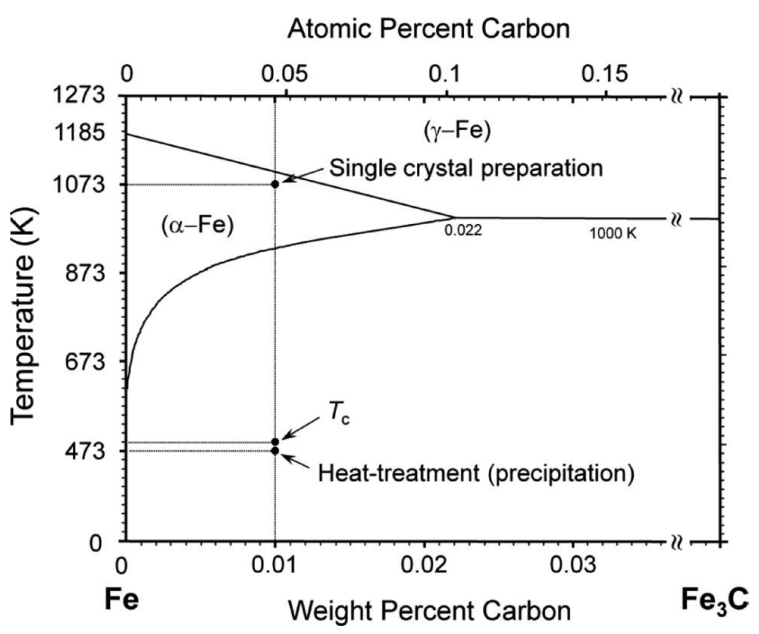

Fig. 2. A phase diagram of the $\mathrm{Fe}-\mathrm{Fe}_{3} \mathrm{C}$ system in the vicinity of a low-carbon region ${ }^{24}$. The temperatures of single crystal growth and precipitation heat-treatment are indicated together with the Curie temperature $\left(T_{\mathrm{c}}\right)$ of cementite.

habit plane is $\{110\} \alpha,{ }^{25,26)}$ each plate appears as a line segment (trace) with a definite orientation in the SEM images. The direction of the trace depends on variant. It is one of the three directions $(\mathrm{A}, \mathrm{B}, \mathrm{C})$ in Fig. 1(b) when observed on the $(1 \overline{1} 0)_{\alpha}$ plane, and one of the four directions $(\mathrm{D}, \mathrm{E}$, F, G) in Fig. 1(c) when observed on the $(001)_{\alpha}$ plane. The correspondence between the trace directions and the variants are summarized in Table 1. In the table, the angle between the field direction and elongated axis $\left(\theta_{1}\right)$ and that between field direction and the easy axis $\left(\theta_{2}\right)$ are also shown. By counting the number of particles having each trace (A to $G$ in Fig. 1), we will be able to understand the influence of shape anisotropy and magnetocrystalline anisotropy on precipitation of cementite.

Figures 3(a) and 4(a) show representative SEM images 
of Specimen-I and Specimen-II, respectively, taken after the precipitation heat-treatment in the absence of a magnetic field. The gray matrix is ferrite ( $\alpha$-phase), and the white elongated particles are cementite. Most of the traces of cementite in Specimen-I are aligned in three directions as indicated by A, B, and C. Those in Specimen-II are aligned in four directions as indicated by D, E, F, and G.

\section{Results and Discussions}

Figures 3(b) and 4(b) show representative SEM images of Specimen-I and Specimen-II, respectively. They are taken after the precipitation heat-treatment under a magnetic field of $7 \mathrm{~T}$. In both specimens, the direction of the trace of the cementite is the same as that formed in the absence of a magnetic field. However, we notice that the amount of precipitate obviously changes after the application of a magnetic field. In Specimen-I, the precipitate with trace B decreases, and in Specimen-II, the precipitate with trace E decreases, whereas that with trace $G$ increases. In order to make a quantitative analysis, we counted the number of precipitates in 24 photographs for each condition, and the average number of particles of cementite with different traces in Specimen-I and Specimen-II are summarized in Figs. 4(c) and 4(d), respectively. The error bars in these figures indicate the standard deviation, and the values within the bracket indicate the fraction (in \%) of particles having each trace. In Specimen-I, the fraction of precipitate with trace B changes from $41 \%$ to $27 \%$ when a magnetic field is applied. In Specimen-II, the fraction of precipitate with trace $\mathrm{E}$ changes from $25 \%$ to $13 \%$, while the fraction of precipitate with trace $\mathrm{G}$ changes from $23 \%$ to $35 \%$ when a magnetic field is applied. Considering the standard deviation, we may regard these changes as significant. This implies that variants selection occurs by the application of magnetic field.

Now we discuss the reason why the fraction of the precipitate changes when a magnetic field is applied. We first consider the influence of magnetic shape anisotropy and
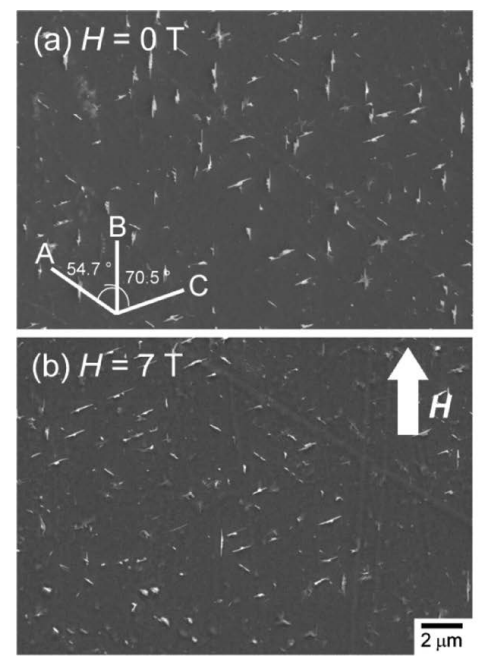

(c) Specimen-I $\left(473 \mathrm{~K}, 24 \mathrm{~h}, \mathrm{H} / /[111]_{\alpha}\right)$

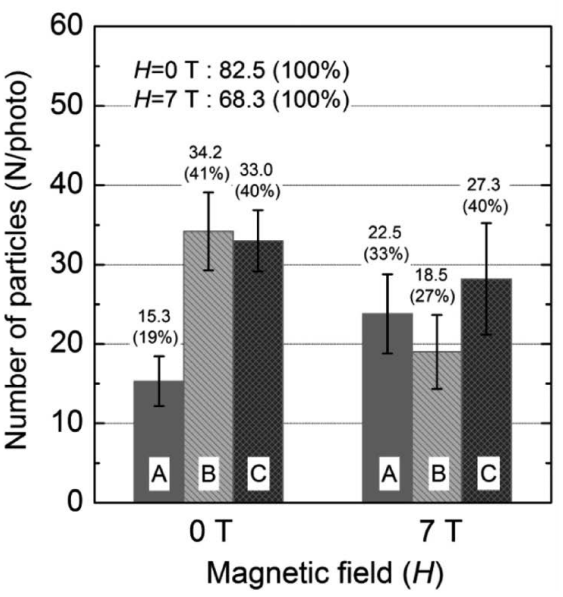

Fig. 3. SEM images of Fe-0.01 mass $\% \mathrm{C}$ alloy after aging heat-treatment under a magnetic field $(\boldsymbol{H})$ of $0 \mathrm{~T}$ (a) and $7 \mathrm{~T}$ (b) applied in the $[111]_{\alpha}$ direction. The bar graph in (c) indicates the average number of particles having traces in the $\mathrm{A}, \mathrm{B}$, and $\mathrm{C}$ directions counted from 30 photographs. The error bar indicates the standard deviation, and the values in brackets indicate the fraction.
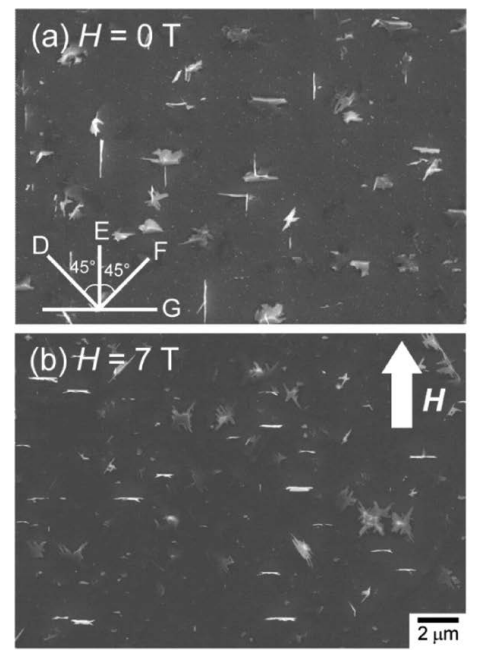

(c) Specimen-II $\left(473 \mathrm{~K}, 30 \mathrm{~h}, \mathrm{H} / /[110]_{\alpha}\right)$

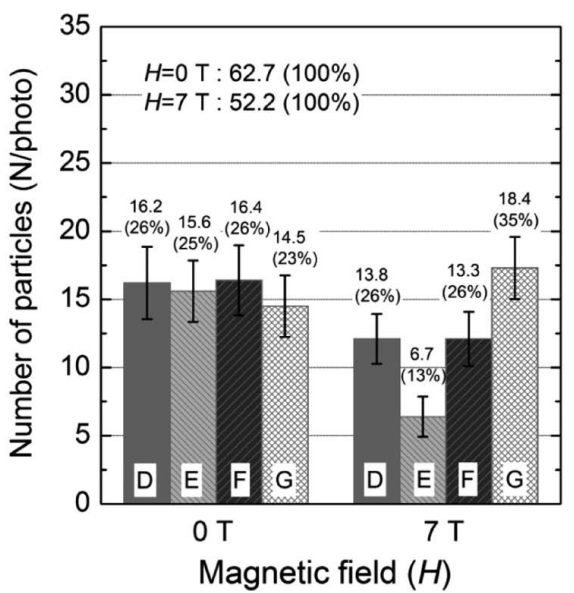

Fig. 4. SEM images of Fe-0.01 mass \% C alloy after aging heat-treatment under magnetic field (H) of $0 \mathrm{~T}$ (a) and $7 \mathrm{~T}$ (b) applied in the $[110]_{\alpha}$ direction. The bar graph in (c) indicates the average number of particles having traces in the D, E, F, and G directions counted from 24 photographs. The error bar indicates the standard deviation, and the values in brackets indicate the fraction. 
then the influence of magnetocrystalline anisotropy.

As shown in Fig. 1, cementite is elongated in the $[100]_{\theta}$ direction. Therefore, there arises shape magnetic anisotropy. The angle $\theta_{1}$ in Table 1 is the angle between the elongated axis of cementite and the field direction $(\boldsymbol{H})$ (see Fig. 1(a)). The shape anisotropy energy increases as $\theta_{1}$ increases for $0^{\circ} \leq \theta_{1} \leq 90^{\circ}$. When the magnetic field is applied in the $[111]_{\alpha}$ direction, the shape anisotropy energy of the V1, V2, and V3 variants is lower than the other variants. Therefore, if the magnetic shape anisotropy were essential for variant selection, the fraction of the V1, V2, and V3 variants would have increased by the application of a magnetic field, and this would have resulted in the increase in the fraction of particles with trace B. This contradicts experimental results, meaning that shape magnetic anisotropy is not essential for variant selection. We also confirm this interpretation as describe below. When the magnetic field is applied in the $[110]_{\alpha}$ direction, the $\mathrm{V} 1, \mathrm{~V} 2, \mathrm{~V} 3, \mathrm{~V} 7, \mathrm{~V} 8$, and $\mathrm{V} 9$ variants have lower shape anisotropy energy than other variants. If the magnetic shape anisotropy were essential for variant selection, the fraction of the V1, V2, V3, V7, V8, and V9 variants would have increased by the application of a magnetic field. This would have resulted in an increase in the fraction of variants with trace $\mathrm{E}$, and a decrease in the fraction of variants with trace $G$. This contradicts experimental results, implying again that shape magnetic anisotropy is not essential for the variant selection.

Next, we consider the influence of the magnetocrystalline anisotropy. The easy axis of magnetization in cementite is $[001]_{\theta}$. The angle $\theta_{2}$ in Table 1 is the angle between the easy axis and the field direction (see Fig. 1(a)). The magnetocrystalline anisotropy energy, $E_{\mathrm{a}}$ increases with increasing $\theta_{2}$ for $0^{\circ} \leq \theta_{2} \leq 90^{\circ}$ as $E_{\mathrm{a}}=K \sin ^{2} \theta_{2}$, where $K$ is the anisotropy constant.

When the magnetic field is applied in the $[111]_{\alpha}$ direction, the V1, V2, V3, V5, and V12 variants have higher magnetocrystalline anisotropy energy than other variants for $\theta_{2}=$ $90^{\circ}$. With the exception of the V1 variant, the trace of these variants is B. Therefore, the decrease in the particles with trace B can be explained by magnetocrystalline anisotropy. When the magnetic field is applied in the $[110]_{\alpha}$ direction, the V1 and V7 variants have the highest magnetocrystalline anisotropy energy for $\theta_{2}=90^{\circ}$. The trace of these variants is E. Whereas, the V4 and V10 variants have the lowest magnetocrystalline anisotropy energy for $\theta_{2}=0^{\circ}$. The trace of these variants is $\mathrm{G}$. Therefore, the decrease in trace $\mathrm{E}$ and increase in trace $G$ after the application of a magnetic field can be explained by magnetocrystalline anisotropy.

From the above discussion, we conclude that the fraction of variants of cementite is essentially influenced by magnetic fields through magnetocrystalline anisotropy. However, in the present study, the experiments were only done at $473 \mathrm{~K}$. If the precipitation is done at elevated temperatures, different results could be obtained since the magnetocrystalline anisotropy energy is generally temperature dependent.

Incidentally, we notice in Figs. 3(c) and 4(c) that the total amount of precipitate decreases after the application of a magnetic field. There are two possible reasons for this behavior. One is a change in the nucleation ratio of cementite caused by the application of a magnetic field. The other is a change in the solubility of carbon in ferrite caused by the application of a magnetic field. A systematic study is necessary to address these problems.

\section{Conclusion}

We found that cementites preferentially precipitate in specific directions from a carbon-supersaturated ferrite by applying a high magnetic field of $7 \mathrm{~T}$. It means that the precipitation of cementite from carbon-supersaturated ferrites is able to be controlled by external magnetic fields which are easily achieved by commercial superconducting magnets.

\section{Acknowledgement}

This work was supported by the Global COE Program "Center of Excellence for Advanced Structural and Functional Materials Design" from MEXT, and by a Grantin-Aid for Scientific Research (C) (24560804) from JSPS.

\section{REFERENCES}

1) V. D. Sadovsky, L. V. Smirnov, Y. Fokina, P. A. Malinen and I. P. Soroskin: Fiz. Met. Metalloved., 27 (1967), 918.

2) T. Kakeshita, T. Saburi, K. Kindo and S. Endo: Phase Trans., 70 (1999), 65.

3) G. Sauthoff and W. Pitsch: Philos. Mag. B, 56 (1987), 471

4) Y. Watanabe, A. Nakano and A. Sato: Mater. Sci. Eng. A, 146 (1991), 151.

5) S. Farjami, M. Yasui, T. Fukuda and T. Kakeshita: Scr. Mater., 58 (2008), 811 .

6) R. Oshima and H. Tokoro: J. Jpn. Inst. Met., 62 (1998), 317.

7) K. Tanaka, T. Ichitsubo and M. Koiwa: Mater. Sci. Eng. A, 312 (2001), 118.

8) S. Farjami, T. Fukuda and T. Kakeshita: Mater. Trans., JIM, 49 (2008), 1970.

9) R. Smoluchowski and R. W. Turner: J. Appl. Phys., 20 (1949), 745.

10) N. Masahashi, M. Matsuo and K. Watanabe: J. Mater. Res., 13 (1998), 457.

11) M. Enomoto, H. Guo, Y. Tazuke, Y. R. Abe and M. Shimotomai: Metall. Mater. Trans. A, 32 (2001), 445.

12) J. Y. Song, X. Zhao, Y. D. Zhang, C. Esling and L. Zuo: Steel Res. Int., 82 (2011), 836.

13) H. D. Joo, S. U. Kim, Y. M. Koo, N. S. Shin and J. K. Choi: Metall. Mater. Trans. A, 35 (2004), 1663.

14) N. Ridley and D. Cheetham: Proc. Inst. Metall. Spring Meet., Pract. Met. Compos., Institute of Metals, London, (1974), D15.

15) X. J. Hao, H. Ohtsuka, P. De Rango and H. Wada: Mater. Trans., 44 (2003), 211.

16) M. Shimotomai, K. Maruta, K. Mine and M. Matsui: Acta Mater., 51 (2003), 2921.

17) X. Hao and H. Ohtsuka: ISIJ Int., 46 (2006), 1271.

18) Y. D. Zhang, C. Esling, M. Calcagnotto, M. L. Gong, X. Zhao and L. Zuo: J. Phys. D: Appl. Phys., 40 (2007), 6501.

19) Y. Xu, H. Ohtsuka and H. Wada: Trans. Mater. Res. Soc. Jpn., 25 (2000), 505.

20) Y. D. Zhang, C. Esling, M. L. Gong, G. Vincent, X. Zhao and L. Zuo: Scr. Mater., 54 (2006), 1897.

21) E. J. Fasiska and G. A. Jeffrey: Acta Cryst., 19 (1965), 463.

22) I. G. Wood, L. Vočadlo, K. S. Knight, D. P. Dobson, W. G. Marshall, G. D. Price and J. Brodholt: J. Appl. Crystallogr., 37 (2004), 82.

23) W. C. Leslie, R. M. Fisher and N. Sen: Acta Metall., 7 (1959), 632.

24) H. J. Choe, T. Terai, I. Miyazaki, S. Yamamoto, M. Yonemura and T. Kakeshita: Abstract of the 150th Academic Meeting (Spring) of the Japan Institute of Metals, JIM, Sendai, (2012), 402.

25) A. Tsuzuki, S. Sago, S.-I. Hirano and S. Naka: J. Mater. Sci., 19 (1984), 2513.

26) E. W. Langer: Met. Sci. J., 2 (1968), 59. 